\title{
Towards UML-Based Formal Specifications of Component-Based Real-Time Software
}

\author{
Vieri Del Bianco ${ }^{1,2}$, Luigi Lavazza ${ }^{1,2}$, Marco Mauri ${ }^{2}$, and Giuseppe Occorso ${ }^{3}$ \\ ${ }^{1}$ Politecnico di Milano, Dipartimento di Elettronica e Informazione \\ P.zza Leonardo Da Vinci, 32, 20133 Milano, Italy \\ \{delbianc, lavazza\} @elet.polimi.it \\ ${ }^{2}$ CEFRIEL, Via Fucini, 2, 20133 Milano, Italy \\ mmauriacefriel.it \\ http://www.cefriel.it \\ ${ }^{3}$ Technology REPLY, Via Ripamonti, 89, 20139 - Milano, Italy \\ g.occorso@reply.it
}

\begin{abstract}
UML-RT is achieving increasing popularity as a modeling language for real-time applications. Unfortunately UML-RT is not formally well defined and it is not well suited for supporting the specification stage: e.g., it does not provide native constructs to represent time and non-determinism. UML+ is an extension of UML that is formally well defined and suitable for expressing the specifications of real-time systems (e.g., the properties of a UML+ model can be formally verified). However, UML+ does not support design and development. This article addresses the translation of UML+ into UML-RT, thus posing the basis for a development framework where UML+ and UML-RT are used together, in order to remove each other's limitations. Specifications are written using UML+, they are verified by means of formal methods, and are then converted in an equivalent UML-RT model that becomes the starting point for the implementation.
\end{abstract}

\section{Introduction}

Formal methods have demonstrated to be effectively applicable in the industrial development of real-time safety critical systems. Nevertheless, formal methods are not widely used in industry. The problem is that while the demand for real-time software increases very fast, the availability of developers who can master formal methods remains little. The consequence is that formal methods are generally considered too difficult or too expensive to be used in "ordinary" real-time software development. On the contrary, UML [14] has achieved a great popularity, essentially because it is a semi-formal notation relatively easy to use and well supported by tools.

Interestingly, UML is gaining popularity also for real-time developments. In fact, UML for Real-Time (alias UML-RT) has been defined on the basis of ROOM [18] and has been rapidly adopted by many developers: it is likely that OMG will include 
UML-RT features in the definition of UML 2.0. However, the application of UML-RT to the real-time domain is still suffering from several problems:

- UML-RT is not formally well defined. This is a relevant limitation of UML-RT, since very often real-time applications are also safety-critical, and thus call for activities like the verification of properties (such as safety, utility, liveness, ...), the simulation of the system, the generation of test cases, etc. It is very hard - if at all possible - to carry out such activities when the specifications are written in semiformal notations like UML or UML-RT.

- UML-RT is an effective notation for the design and implementation of systems, but not very well suited for representing requirements or specifications. For instance, when modeling the environment in which a real-time system has to work it is often necessary to represent non-deterministic behavior or simultaneous events. These phenomena are not supported by UML-RT.

- Finally, time issues (i.e., the representation of time and time constraints) are not treated at a native level: ad-hoc components (like timers) have to provide timerelated information to the system. This is not generally perceived as a big problem at the design level, as designers consider quite natural to model the existence of timers and similar objects.

In previous work we addressed some of the above problems. In fact we adopted a dual language approach to real-time software development: in a first phase models are written in UML according to the usual modeling practices; in a second phase UML models are automatically translated into one or more formal notations, which provide support to activities such as the simulation, the verification of properties, the generation of test cases, etc. In this way, developers exploit the advantages of formal notations while skipping the complex and expensive formal modeling phase, since they can use the notation they are familiar with.

Actually we had to extend UML in order to let it satisfactorily specify real-time systems and to provide it with formal semantics $[11,5,7,6]$. The models written in the resulting language (called UML+ throughout this paper) can be automatically translated into equivalent TRIO temporal logic formulas [9] or into timed automata [1]. In this way existing formal methods can be applied. For instance the properties of the model can be verified by means of the Kronos model checker [20].

Although such work demonstrated the viability of the approach, our approach did not support the design and implementation activities. Consistently with our goal of defining a development method that can be effectively applied in industrial settings, we chose UML-RT as a target language. The reasons for this choice are that UML-RT is a very good notation for component-based developments, it is going to become a standard (it is very likely that it will be included in UML 2.0) and it is already very popular in industry. The aim of the work reported here is thus to build a bridge between our specification-oriented UML+ and the design-oriented UML-RT.

The paper is structured as follows. Section 2 provides a brief introduction to UML+. Section 3 briefly recalls the main characteristics of UML-RT. Section 4 illustrates the problems for translating UML+ models into UML-RT and describes the proposed solutions. Section 5 describes the development environment that implements 
the proposed approach. Section 6 presents a simple case study as a proof of concepts. Finally, Sect. 7 draws some conclusions and briefly accounts for related work.

\section{A Brief Introduction to UML+}

The semantics associated with UML+ is directly inspired by the Timed Statecharts defined by Kesten and Pnueli [11]. This formalism extends the traditional statecharts by specifying time limits for the execution of transitions. The semantics is defined with reference to a dense time domain. This implies that the system may deal with events that are arbitrarily close in time to each other. Transitions are classified in two types: immediate ones and timed, or waiting, transitions. Immediate transitions do not depend on time: they are executed when a triggering event occurs. When no immediate transition is enabled, the time can flow with the state of the system remaining unchanged. On the contrary, timed transitions are independent from events. They are associated with a time interval that specifies a minimum and a maximum waiting time: the transition cannot be executed before the minimum or after the maximum waiting time. If no event changes the current state, the timed transition must be executed before the maximum waiting time. In Timed Statecharts negated events can appear in the conditions which guard the execution of transitions. The concept of "step" is associated with the execution of an immediate transition; a reaction to an event may occur several steps after its generation, but still in the same timestamp. This kind of semantics is based on the fact that every generated event "persists" until the time does not flow. The time may flow only if all the transitions which were enabled by that event have been executed. In this way, several transitions triggered by the same event $e$ are executed before the time becomes grater than the time of the occurrence of $e$.

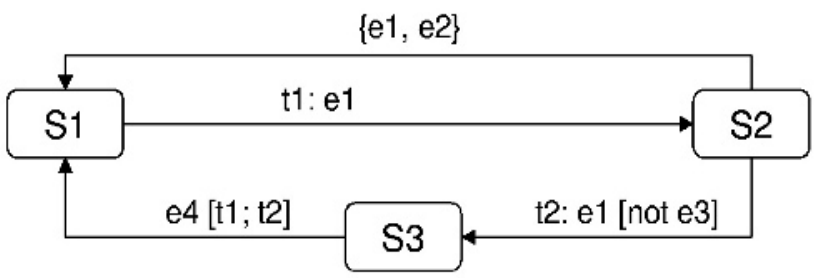

Fig. 1. A UML+ statechart

Timed Statecharts have been further extended in UML+ to accommodate most of the syntax of UML State Diagrams that is not present in Timed Statecharts; for instance, inter-level transitions and fork transitions are allowed in UML+. In UML+ it also possible to associate transitions with both events and time intervals (see for instance the transition from S3 to S1 in Fig. 1).

UML+ allows the modeler to associate a set of events to a transition, indicating that the transition is triggered by the concurrent occurrence of the set of events (see the transition from S2 to S1 in Fig. 1). 
In UML+ guards can make reference to events. It is possible, for instance, to specify that a given transition is executed if event $e l$ occurs while event $e 3$ is not occurring (see the transition from S2 to S3 in Fig. 1).

Besides the modification of statecharts, UML+ does not exhibit differences with respect to standard UML. However, our approach to verification of models based on UML+ only takes into account class diagrams, state diagrams and object diagrams. Other diagrams are ignored if present. The elements of the class diagrams are employed with their usual role and meaning. However, it must be noted that - being our approach devoted to the verification of real-time properties only - the attributes and the methods that do not affect real-time behavior of the models are ignored.

A detailed definition of UML+ can be found in [12,5,7].

\section{A Brief Introduction to UML-RT}

UML-RT is an extension of UML that addresses real-time issues. It provides a formalism to handle active objects. An active object is called a Capsule in UML-RT and it communicates with other capsules through asynchronous messages, which are sent and received through Ports. A Port is defined by a Protocol that defines which messages can be sent through a port (Out messages) and which messages a port accepts (In messages). Given a Protocol, its conjugate is always defined by simply inverting the In and Out messages.

The State Diagrams associated to each capsule have the usual syntax and semantic as in plain UML, including the "run to completion" behavior [14]. The only additional constraint is that any message (except internal messages, which remain in the boundaries of the State Diagram) is always associated with a Port.
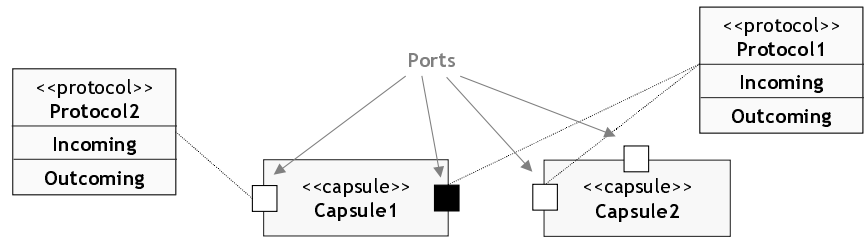

Fig. 2. Capsules and ports in UML-RT

Capsules are connected through Connectors. A Connector binds two different Ports with compatible Protocols. A protocol is always compatible with its conjugate. Moreover, a protocol $\mathcal{P} 1$ is compatible with another protocol $\mathcal{P} 2$ if $\mathcal{P} 1$ accept as In messages a superset of the $\mathcal{P} 2$ 's Out messages and the set of $\mathcal{P} 1$ 's Out messages is a subset of the messages accepted by $\mathcal{P} 2$. 


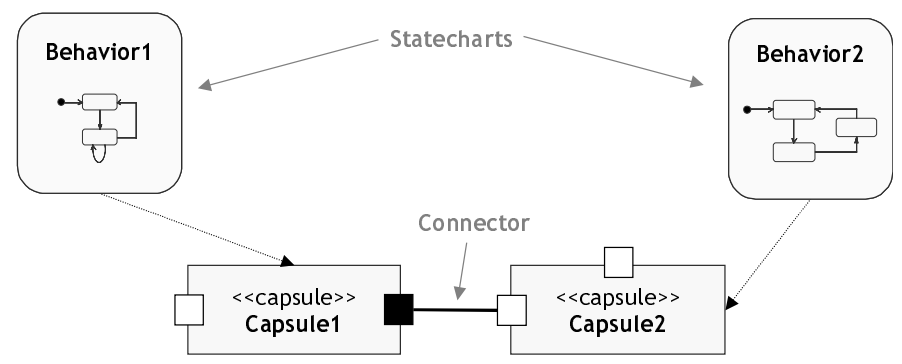

Fig. 3. Connectors and state diagrams in UML-RT

UML-RT mainly focuses on the concept of active component (the Capsule) and does not directly address real-time constraints. The concept of time can be found in standard UML-RT libraries - mainly thanks to the Timer class stereotype- but not directly in the modeling language.

UML-RT is implementation-oriented: it is conceived to be used with a complete library in a language of choice, usually abstracting form the underneath platform. By embedding code fragments in transitions and states (as defined in plain UML) a UMLRT model can be directly translated into code (Rational Rose RealTime being the reference tool for generating working embedded distributed systems from UML-RT models).

\section{From UML+ to UML-RT}

UML+ is a formal notation to express the desired behavior and the constraints of realtime systems; i.e., it can be used to formalize real-time requirements. UML+ models are written in a visual language very close to standard UML 1.4, and can be translated in a formal language and checked to verify that the systems behave as required and that the constraints are satisfied.

\subsection{A Case Study}

In the rest of the paper an example is used to illustrate the proposed approach. The system to be modeled and developed is the Generalized Railroad Crossing (GRC) [10], one of the best known benchmarks proposed in the literature for evaluating formalisms and tools dealing with real-time software.

The system to be modeled operates a gate at a railroad crossing. The railroad crossing I lies in a region of interest $\mathrm{R}$ (see Fig. 4). Trains travel through $\mathrm{R}$ on $\mathrm{K}$ tracks in one direction (having trains traveling in both directions does not change the complexity and 


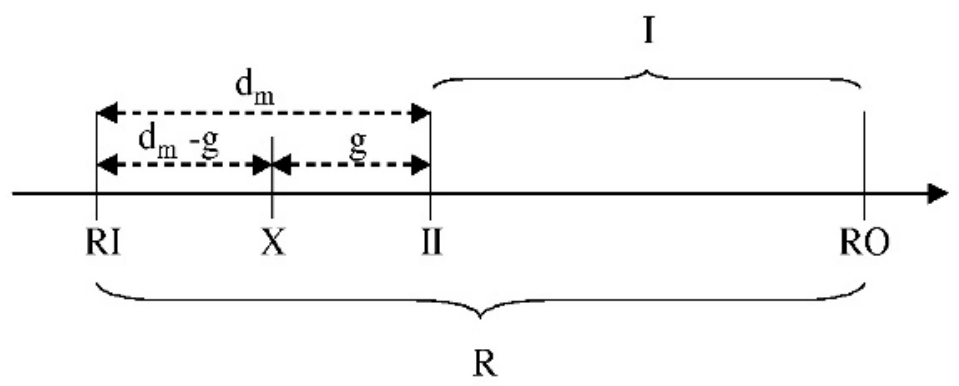

Fig. 4. GRC regions of interest

relevance of the case study). Trains can proceed at different speeds, and can even pass each other. Only one train per track is allowed to be in R at any moment. Sensors indicate when each train enters and exits regions R and I. Point RI and RO indicate the position of the entrance and exit sensors for region R. II indicates the position of the sensor which detects trains entering region I. $\mathrm{dm}$ and $\mathrm{dM}$ are the minimum and maximum time taken by a train to cross RI-II zone. $\mathrm{hm}$ and $\mathrm{hM}$ are the minimum and maximum time taken by a train to cross zone $\mathrm{I}$. $\mathrm{g}$ is the time taken by the bars of the gate to move from the completely open to completely closed position.

\subsection{UML+-RT: Making UML+ Component-Aware}

In order to support component-based development we have to make UML+ component-aware. For this purpose we adopt the representation of components proposed by UML-RT, i.e., the capsules. This choice was taken because capsules are a satisfactory formalism, and because in this way it is easier to convert UML+ models into UMLRT models. The result of merging UML+ (featuring the Timed Statecharts [11]) and UML-RT is a new modeling language called UML+-RT.

UML+ specifications of real-time system are essentially composed of class diagrams and object diagrams; the instances of classes are active objects and their behavior is defined by the associated Timed Statecharts. The structure of the system is defined by an Object Diagram that is used to connect the class instances that compose the system. In UML+-RT we retain the same organization. As an example, let us consider the model of the GRC. Figure 5 illustrates the class diagram of the system, where capsules, ports and protocols are explicitly modeled. The object diagram is used to define the first level capsule. This is relatively straightforward, since the Component Diagram of UML-RT is a specialization of the UML Collaboration Diagram [16,18], as are the object diagrams. Figure 6 illustrates the structure of the railroad crossing system in terms of capsules and connections, highlighting the connections to ports. 


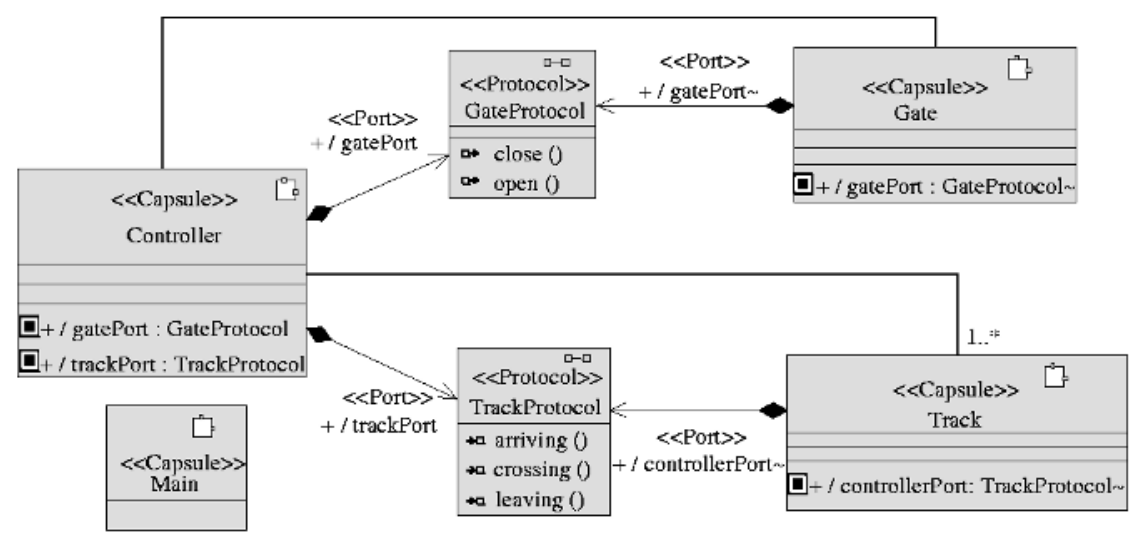

Fig. 5. UML+-RT class diagram

$$
\begin{aligned}
& \text { /gate: Gate + / gatePort + / gatePort / controller: } \\
& \text { : GateProtocol : GateProtocol Controller } \\
& \text { +/ controllerPort: TrackProtocol } \\
& \text { / track: Track }{ }^{\mid \text {III }} \text { TrackProtocol }
\end{aligned}
$$

Fig. 6. Main capsule: UML+-RT component diagram

The behavior of a Capsule is defined by the associated Timed Statechart. For instance, Fig. 7 describes the behavior of class Gate: it clearly indicates that the events which cause the state transitions are received through the gatePort.

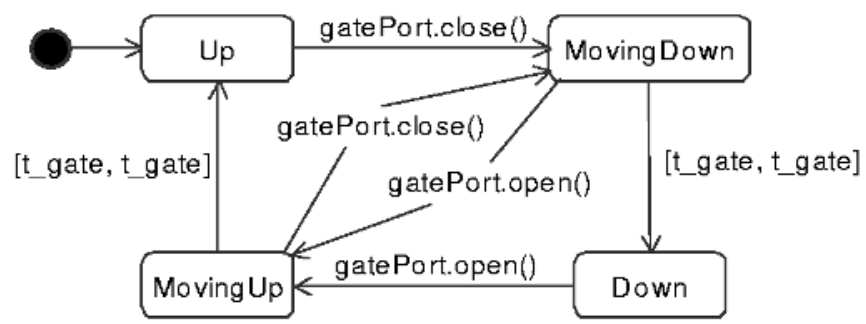

Fig. 7. Timed statechart for the Gate class 


\subsection{Dealing with Synchronous Semantics}

UML+ adopts the synchronous semantics of Timed Statecharts. Synchronous semantics - although useful in the analysis phase and ideal for the verification by model checkers - is unrealistic in the implementation phase. In a real system there are no cheap and easy ways to verify that two events are concurrent, to make two transitions fire simultaneously, and when the system is distributed it is not even easy to process messages in the same order in which they were generated. On the contrary, systems are implemented according to an asynchronous semantics. Most of the modeling tools that generate an implementation are based on asynchronous models. Hence it would be desirable to be able to transform UML+ synchronous specifications into asynchronous models, like UML-RT models. UML-RT was chosen as the target notation because it is probably going to become a standard, it is component-oriented (an important feature to facilitate reuse), and it is equipped with tools (one for all, Rational Rose RealTime) to generate working systems.

The translation from UML+-RT to UML-RT is not simple. The "run to completion" semantics of the UML-RT State Diagrams is quite different from the "synchronous execution of transitions" semantics of Timed Statecharts adopted in UML+ and UML+-RT. This difference can lead to some hard problems, described in Sect. 4.4. The problems that can arise are of two types:

- Some model fragments simply do not make sense in UML-RT. For instance, in UML-RT it is not possible to deal with negated events or to associate a transition with a set of simultaneous events.

- Some models have the same general meaning in UML-RT as in UML+, but their behavior is not actually the same.

In the first case it is difficult to devise which UML-RT model would more closely represent the intended meaning of the given UML+ model. Therefore we decided not to translate models having this kind of characteristics, i.e., it is responsibility of the modeler to correct these situations.

In the second case the model interpreted according to the UML-RT semantics could violate the properties that were proved valid for the same model interpreted according to UML+ semantics. In this case the modeler is invited to build a second, more realistic UML+ model that takes into account the execution environment, and is therefore able to predict whether properties will remain valid also in the implementationoriented UML-RT model.

The next section describes the problems mentioned above (and how to deal with them).

\subsection{Issues and Problems with the Translation from UML+-RT to UML-RT}

Consider the transition from $\mathrm{S} 2$ to $\mathrm{S} 1$ and the transition from $\mathrm{S} 2$ to $\mathrm{S} 3$ in Fig. 1: managing concurrent events and negated events in UML-RT is impossible, therefore in 
these cases the translator issues a warning and does not produce any UML-RT model. It is the modeler who has the responsibility to produce a more realistic model having similar characteristics. For instance the transition from S2 to S1 could be modified as shown in Fig. 8.

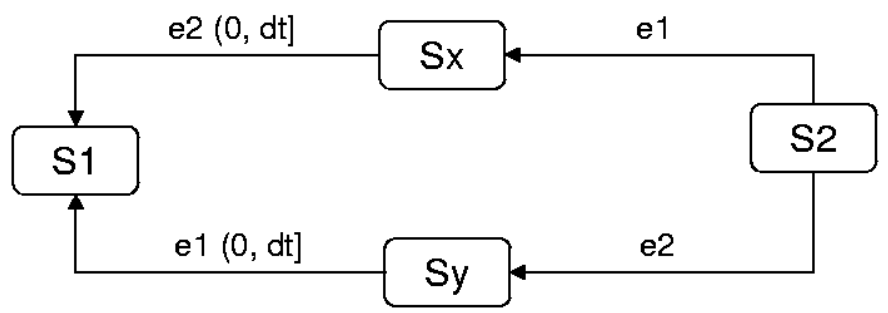

Fig. 8. A statechart handling "almost concurrent" events

The statechart in Fig. 8 prescribes that the transition from S2 to S1 happens when events e 1 and e 2 occur (in any order) in a [0, dt] interval. If $\mathrm{dt}$ is little the transition will happen when e 1 and e 2 occur in a very short - though finite - interval, while the original condition (given in Fig. 1) required that e1 and e2 occurred exactly at the same time. In practice there is generally no difference between the two specifications. In any case it is always possible to model-check the new version of the model containing the transition from S2 to S1 redefined as shown in Fig. 8, in order to assure that the desired properties still hold.

Transitions involving negated events, timed transitions having $\mathrm{t}_{\min }=\mathrm{t}_{\max }$ (i.e., transitions reacting to events that must occur at a precise time) and instantaneous transitions (i.e., transitions that take null time to execute, even though they are associated with some action), can be treated in a similar way. In particular, we can specify that two events do not occur in a short finite interval, or that an event occurs in a short finite interval, or that an action takes a short finite time to complete.
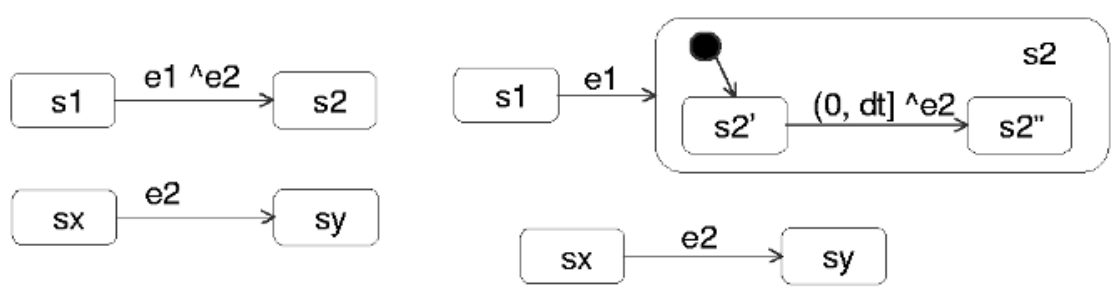

Fig. 9. A model containing simultaneous events (left) and the equivalent model with no simultaneous events

Timed transitions, i.e., transitions bounded with a time interval $\left[\mathrm{t}_{\min }, \mathrm{t}_{\max }\right]$, are easily represented in UML-RT when $t_{\max }>t_{\min }$. When a state having one or more of such outgoing timed transitions is entered clocks are set (via the UML-RT libraries) to $t_{\min }$. When the state completes its activities (if any), it waits the expiration of the appropriate clock before evaluating the guards associated with the timed transitions, and finally 
fires one of the enabled transitions. Clocks can be set also on the upper bound $\mathrm{t}_{\max }$. In this case, if the state completes its activities after the upper bound, an exception is raised, reporting that the state activities have not respected the timing constraints specified in the original UML+-RT model. These exceptions are useful for testing; in any case it is a design decision how to handle them, i.e., whether to execute a transition even if the upper bound time has passed or to take a different action.

Now let us consider the statechart reported in the left part of Fig. 9. In this case the statechart has a well defined meaning in UML-RT, but unfortunately the behavior of the system in UML-RT is not the same as in UML+. In fact in UML+ events e1 and e2 occur at the same time (i.e., in the same timestamp), while in UML-RT e2 will follow e1 with a finite (though probably little) delay, since events are extracted from the event queue one at a time. This means that the properties that hold for the UML+ model could be violated by the same model behaving according to UML-RT semantics.

In these cases we have models that are easily translated into UML-RT, but whose behavior will not match the previously model-checked one. We decided to solve this problem by maintaining two models: one for the purpose of translation, and another for the purpose of model checking (see Sect. 5). The latter model must reflect the behavior of the target UML-RT model. This can be achieved in two ways:

1. The modeler explicitly represents that transitions take a non-null time, e.g., by assigning a positive lower bound to the time intervals associated with the transitions. For instance, the UML+ model reported in the right part of Fig. 9 behaves as the UML-RT model reported in the left part of the same figure: event e 2 follows event 1 after a finite time (not grater than $\mathrm{dt}$ ).

2. The modeler explicitly models in UML+ the event queue that is implicitly assumed by UML-RT statecharts. This requires to set the maximum size of the queue.

In both cases the size of the model increases very fast as the number of events that have to be taken into account in the realistic delay period increases.

Figure 10 illustrates a model exploiting a queue. The queue is a FIFO container that is loaded with the arriving events and releases them in a finite time, which represents the time actually needed to perform a transition. In the left part of Fig. 10 when e1 (or e4) occur, instead of issuing the events e 2 (or e3) which would imply an instantaneous transition to state sy (or sz), the events e $2_{\mathrm{q}}$ (or e $3_{\mathrm{q}}$ ) are issued. These events are "captured" by the queue (right part of Fig. 10), which will issue the event e2 (or e3) after a finite delay $\leq \mathrm{dt}$, thus causing the transition to sy (or sz). Note that here we made two assumptions:

- The maximum time to handle a transition is dt. Therefore the transition is associated with a time interval $(0, \mathrm{dt}]$. If a minimum time $\mathrm{dt} \_$min were also defined the time interval would be [dt_min,dt].

- The length of the queue is 1 . In general the length of the queue is given by the maximum number of events that can arrive during time interval $[0, \mathrm{dt}]$. In the case depicted in Fig. 10 e 2 and e 3 are considered mutually exclusive (at least in the considered situation, i.e., immediately after the transition from s1 to s2 or s3). 

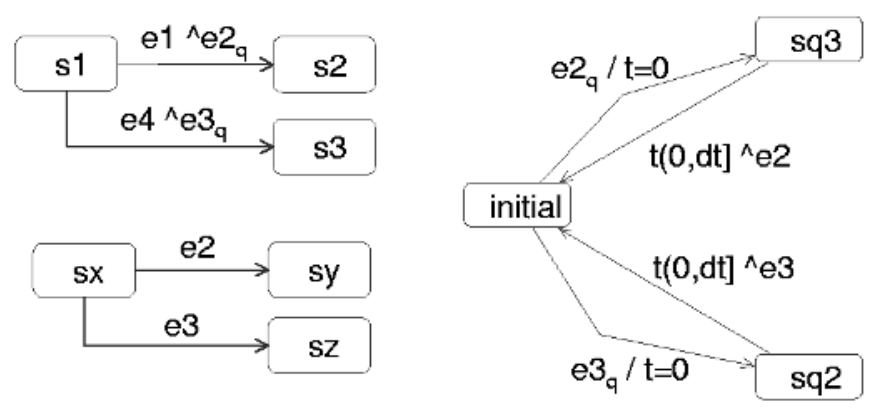

Fig. 10. A model featuring a queue

In Fig. 10 the notation $t(0-\mathrm{dt}]$ associated with a transition indicates that the transition has to occur in the interval $(t, t+d t]$. The notation $t=0$ indicates that clock $t$ is reset. In this simple case this notation is actually not necessary (since $t$ is always reset when exiting from state initial, $t(0-d t]$ is equivalent to $(0-d t]$ in the transitions from sq2 and sq3 towards initial). However, in longer queues it is necessary to use this notation to express properly the time constraints.

\section{The Development Environment}

Thanks to the adoption of Timed Statecharts, UML+-RT has well-defined semantics. This allowed us to write a program that translates UML+-RT models into timed automata, so that the model checking tool Kronos [20] can be used to verify properties [3]. This is sufficient to support the specification phase, but does not help much in the design and implementation phase. For this purpose we want to generate a UML-RT model that preserves the properties already formally verified. However, the original model has to be modified, since it features perfect concurrency or synchronicity, which cannot be generally implemented in a model featuring asynchronous semantics. The problem is to preserve the properties of the original model, but also to check that these properties do not rely on characteristics of the model that cannot be implemented in the real world. Our approach is to make incremental refinements of a UML+-RT model until we achieve a model that can be safely translated into UML-RT. At first sight, this approach could seem to be a not very good trade-off, as long as most of the difficulties of the conversion are left with the modeler. However we believe that in this way some relevant advantages are achieved:

- the modeler is guaranteed that the properties of the original model are maintained;

- the modeler is guided in the modification of the model by the diagnostic messages provided by the translator;

- the modeler achieves a deeper understanding of the model, as new details (like the realistic duration of a transition) are added;

- the final model actually reflects the ideas of the modeler. 


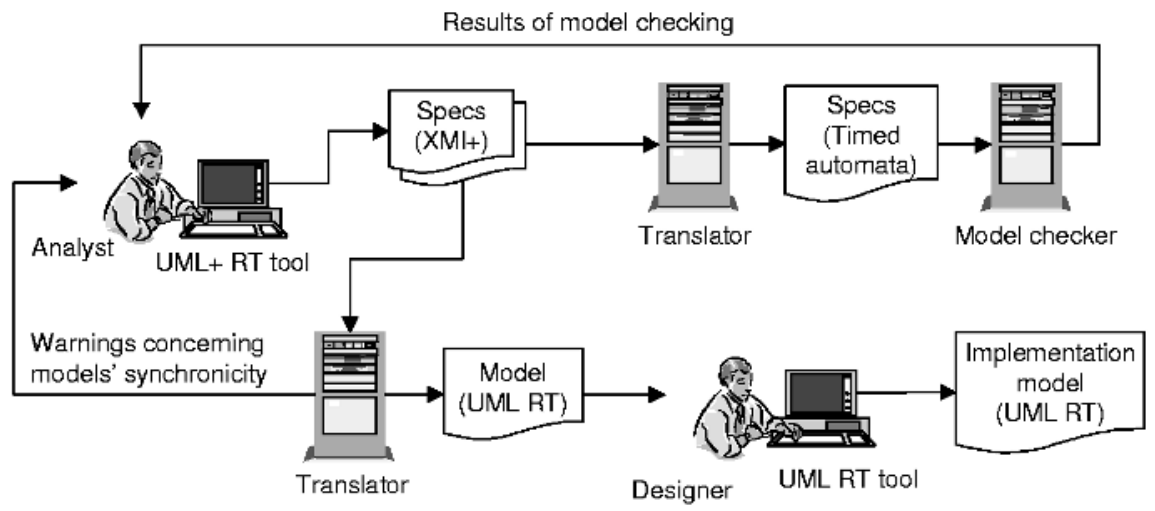

Fig. 11. The envisaged programming environment

Figure 11 illustrates the programming environment that implements the proposed approach. This environment supports a development process organized as follows.

Initially the modeler creates a UML+-RT model without worrying about the implementation of the model. The product of this phase is an "ideal" model, whose properties are verified by means of the model checker.

The ideal model is then processed by the translator, which tries the conversion into UML-RT. In general the translator will find problems with the synchronicity of the model (see Sect.4.4) that are notified to the modeler. The latter modifies the model in order to remove the most obvious problems (simultaneous events, negated events, etc.). The new model is again verified by means of the model checker.

The resulting "more realistic" model $\mathrm{M}$ is finally translated into a UML-RT model $\mathrm{M}_{\mathrm{RT}}$. This step is mainly devoted to replacing timed transitions with transitions triggered by events generated by timers; the setting, resetting, etc. of timers is determined by the translator on the basis of the timed transitions. However, $\mathrm{M}_{\mathrm{RT}}$ will not behave exactly like $\mathrm{M}$, e.g., because in $\mathrm{M}$ transitions are instantaneous, while in $\mathrm{M}_{\mathrm{RT}}$ they take a finite time. In order to verify the properties of $\mathrm{M}_{\mathrm{RT}}$ the modeler builds a second UML+-RT model M', which modifies $\mathrm{M}$ in order to represent its behavior according to the rules of the UML-RT environment (e.g., by introducing suitable queues of events). In practice in this phase the modeler maintains two models: $M$ ' for the purpose of model checking, and $\mathrm{M}$ for the purpose of translation into $\mathrm{M}_{\mathrm{RT}}$. The behavior of $\mathrm{M}_{\mathrm{RT}}$ in the UML-RT environment is equivalent to the behavior of $\mathrm{M}^{\prime}$ in the synchronous model-checking environment. This equivalence descends from the fact that the modeler built $\mathrm{M}$ ' as a version of $\mathrm{M}$ where the "non-ideality" of the execution environment is adequately considered. We considered the automatic construction of $\mathrm{M}$ ' too complex and/or too impractical to be attempted. The result is that the responsibility of maintaining $\mathrm{M}$ and $\mathrm{M}$ ' equivalent remains with the modeler. Generally, he/she is in a very good position to judge which features of the model $\mathrm{M}$ are too idealized, and, in these cases what are the sufficiently realistic corresponding models. For instance, the modeler can evaluate if a delay in handling a signal is long enough to alter the behavior of the system, and - if so - to explicitly model that delay (note: a prudent approach is to model the delay, and then remove it if the behavior is not affected). 
At the end of this process, $\mathrm{M}_{\mathrm{RT}}$ is the starting point for the coding and testing phases. In UML-RT it is still possible to refine the model, but the refinements should be careful to preserve the properties that were successfully checked (the verification of the properties of component-based implementations in known run-time environments is the subject of an ongoing research activity).

As a final observation, it should be noted that UML-RT is also used for introducing components in UML-based development of non real-time software in a more effective way than using standard UML components in deployment diagrams. Our approach is not very effective for this kind of developments. In fact, although it is possible to build UML+ (and UML+-RT) models of any system, in general systems that do not exhibit a real-time behavior do not need to be translated and model-checked as discussed above.

\section{Validation}

The system described in Sect. 4.1 has to operate the crossing gate in a way that satisfies the following two properties:

Safety: The gate is closed during all occupancy intervals.

Utility: If no train is in any occupancy interval, nor within $\xi 1$ prior to an occupancy interval, nor within $\xi 2$ after an occupancy interval, then the gate is open.

Point X (see Fig. 4) is thus defined as follows: when a train enters zone X-II it is time to start closing the gate, so that the bar will be completely lowered when the train arrives at II. The exact position of $\mathrm{X}$ depends on the speed of the fastest trains. In order to have the gate closed when the fastest trains arrive at II, we must begin to close the gate dm-g time units after the train entered region $\mathrm{R}$. If the train is slower the gate will be already closed when it enters the crossing region I.

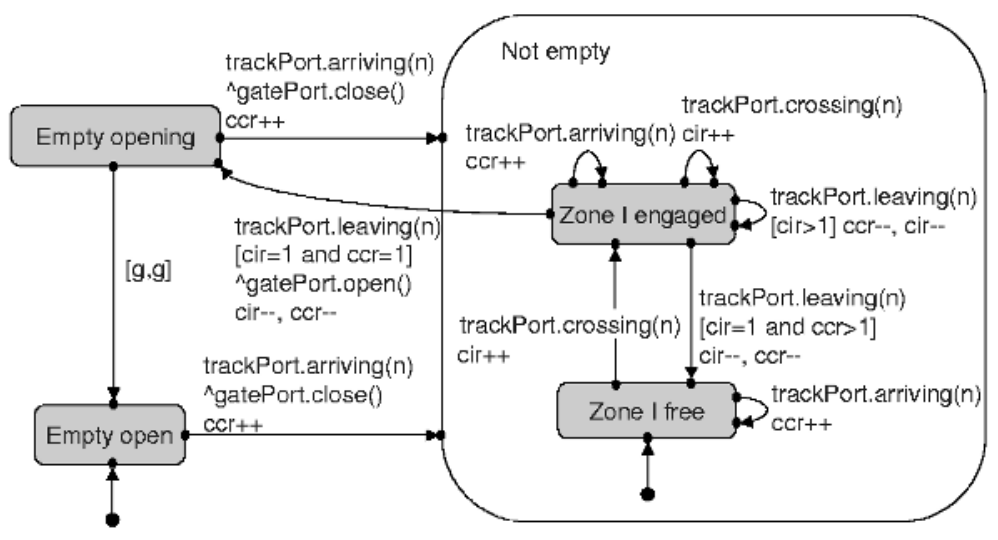

Fig. 12. The statechart of capsule controller

The behavior of the controller is defined as specified in Fig. 12. The idea is that the Controller counts the trains in the X-RO zone (by means of variable $c c r$ ) and in the II- 
RO zone (by means of variable $c i r$ ). As soon as $c c r$ becomes grater than zero the controller sends the close command to the gate. When $\mathrm{ccr}$ and $\mathrm{cir}$ become zero the open command is sent to the gate. The signals crossing and leaving correspond to the signals generated by the sensors II and RO, while signal arriving corresponding to point $\mathrm{X}$ must be delayed of dm-g time units with respect to the signal generated by sensor RI. It is responsibility of the Track components (whose statecharts are omitted because of space reasons) to satisfy these rules. The parameter $n$ of the arriving, crossing and leaving events is the identifier of the track which issues the signal.

An important observation: the statechart given in Fig. 12 cannot be analyzed by Kronos as it is, because integer variables (like $c c r$ and $c i r$ ) are not allowed in the timed automata analyzed by Kronos. Since the number of tracks is limited this problem can be easily solved by replacing the integer counters with finite state automata where each state represents a value. Moreover, the events cannot be parameterized: in the Kronos model $\operatorname{arriving}(n)$ must be replaced by arriving1, arriving2, ..., arrivingN (where $\mathrm{N}$ is the number of tracks). This applies of course to crossing and leaving events as well.

The model of the GRC described above was translated into a set of timed automata that satisfies the required properties (this was proven by means of the Kronos model checker).

The model is relatively simple, thus it can be directly translated into a UML-RT model, but the latter would not always behave as the original UML+-RT model. In fact by applying our translator (which implements the concepts described in section 4) we obtain a set of warnings, indicating that a new model is needed to verify the actual behavior of the UML-RT system. The warnings by the translator concern the following issues:

- In the statechart of capsule Gate the reactions to events open and close are immediate. We need a queue to simulate non instantaneous transactions: in this case a 2 events, 2 place queue. The distance between an open and a close event is no less than $\mathrm{g}+\mathrm{hm}$ (due to the behavior of the Controller). Being dt1 the time to handle one event, it must be $2 \mathrm{dt} 1<(\mathrm{hm}+\mathrm{g})$. For a real computer-based system this condition is very easily satisfied.

- In the statechart of capsules CounterCir and CounterCcr (not shown) there are instantaneous transactions. In the worst case, $N$ fastest trains enter region $R$ simultaneously: they will enter region X-II simultaneously, they will enter region II-RO simultaneously and finally they will exit region I simultaneously. We need 2 queues to manage these simultaneous transitions: one for CounterCir and one for CounterCcr. In both cases two pairs of events are relevant: arriving-leaving for CounterCcr and crossing-leaving for CounterCir. Both the queues are organized as follows ( $\mathrm{N}$ being the number of tracks). The queues are a 2 events $2 \mathrm{~N}$ place queue. Being dt2 the time taken to process a single event, it must be $(2 \mathrm{~N}$ $d t 2)<(d m+h m)$. In fact, in the worst case, $N$ fastest trains entering simultaneously fill the queue with $2 N$ events, which must be handled in no more than $(d m+h m)$ time units (i.e., before all the trains leave the crossing). For a real computer-based system this condition is very easily satisfied.

- Capsule Track generates signal arriving exactly $d m-g$ time units after receiving the signal generated by sensor RI. Signal arriving is communicated instantly to 
the Controller. This is unfeasible in a real-life environment. We have to give time to a real-world controller to react: for this purpose we specify that signal arriving is issued $(d m-g)-d \_r e a c t$ time units after the signal from the sensor is received, where $d \_$react is the time taken by the real environment to react to an event (including event handling, transmission, etc.).

Following the principles illustrated in Sect. 5 we built a "more realistic" UML+-RT model. This model was then translated into UML-RT, and the resulting model was tested by means of AnyLogic, a tool that provides a simulation environment for UMLRT models. The simulation showed that the system actually behaves as required. The simulation can be seen at http://www.xjtek.com/applications/?area=traffic (the model can be downloaded from the same site). Of course the simulation does not guarantee that sooner or later an erroneous situation will occur. In order to exclude this possibility we need to formally prove the properties of the system. For this purpose, we also built -following the indications of the translator reported above- the model that reproduces the behavior of the environment. This model - translated into timed automata and checked by means of Kronos - showed that the system's behavior actually satisfies the requirements. Note that in order to satisfy the requirements, $d$ _react has to be given a proper value. Such value can be computed taking into account the delays introduced by the queues and the characteristics of the model or - more simply - by means of a trial-and-error process. In fact by running Kronos with half a dozen different values of $d \_$react we were able not only to find safe values, but also the minimum safe value.

The Kronos model was also used to find unsafe working conditions for the system (e.g., dm too short with respect to the speed of the gate). Modifying the simulation model accordingly we were able to simulate with AnyLogic the occurrence of erroneous situations.

\section{Conclusions and Related Work}

Several research activities were carried out in order to provide UML with formal semantics. However, such initiatives assume perfect technology (like [8]) or are oriented to providing UML with a precise underlying model (like [17]).

In fact, one of the main obstacles to the application of rigorous development techniques is the difference between real-time application software and functional design (which adopt simplifying assumptions, like instantaneous and perfect communications, synchrony of interaction with the environment, or atomicity of actions) and the physical real-time systems [19].

In order to solve the "synchrony assumption" Taxys provides a compiler that derives a verifiable model from programs written in Esterel and C [2]. In particular, the model includes the specification of an "event handler" that represents the interface between the external environment and the real-time application (and plays a role similar to our event queues). 
The work presented here is an initial effort to tackle the problem of bridging a perfect, synchronous, specification-oriented UML-based formalism with the real, imperfect, asynchronous implementation world. In particular we addressed the conversion of UML+ (an extension of UML that is suitable for the specification of real-time systems [5]) into a notation suitable for implementation, namely UML-RT, with the constraint that the final model retains the properties of the original model. This goal required to add the concept of component (or capsule) to UML+, and to map the synchronous semantics of UML+ onto the asynchronous semantics of UML-RT. The first task was easily solved by borrowing the component notation of UML-RT. The graphical language obtained (called UML+-RT) integrates the component-oriented concepts of UML-RT with the elements of UML+, and specially with Timed Statecharts [11]. Much harder is the conversion of a synchronous model into an asynchronous one. We propose a translation process based on incremental refinements of the original UML+RT model, guided by the translation tool and aiming at the complete removal of the features that model "ideal" situations that cannot be achieved in practice. In particular we propose to use two models, one to be translated into UML-RT for implementation, and another explicitly modeling the mechanisms of the real execution environment, in order to formally prove the properties of the system in real working conditions.

Figure 11 illustrates the development environment which exploits UML+-RT and the associated tools. The construction of the tools is complete, while their integration is in progress. The environment will enable a development process where the development is mainly based on model construction, and the relevant properties of the models can be checked step-by-step.

Acknowledgments. The work described here was carried out as part of the ITEA project DESS (Software Development Process for Real-Time Embedded Software Systems). Project DESS is partly supported by MIUR. More on DESS can be found at http: / / www. dess-itea.org.

Giuseppe Occorso was with CEFRIEL when he participated in the work reported here.

\section{References}

1. Alur, R., Dill, D.L.: A Theory of Timed Automata. Theoretical Computer Science, n.126 (1994) 183-235

2. Bertin, V., Closse, E., Poize, M., Pulou, J., Sifakis, J., Venier, P., Weil, D., Yovine, S.: Taxys $=$ Esterel + Kronos. A tool for verifying real-time properties of embedded systems. Conference on Decision and Control, CDC'01. Orlando, IEEE Control Systems Society (2001)

3. del Bianco, V., Lavazza, L., Mauri, M.: An application of the DESS modeling approach: The Car Speed Regulator, In Proc. SIVOOES 2001, Budapest (2001)

4. del Bianco, V., Lavazza, L., Mauri, M.: A classification of real-time specifications complexity, In Proc. SIVOOES 2001, Budapest (2001) 
5. del Bianco, V., Lavazza, L., Mauri, M.: An introduction to the DESS approach to the specification of real-time software. CEFRIEL Technical Report RT01002 (2001)

6. del Bianco, V., Lavazza, L., Mauri, M.: Model Checking UML Specifications of Real Time Software. The Eighth IEEE International Conference on Engineering of Complex Computer Systems (ICECCS02), Greenbelt (2002)

7. del Bianco, V., Lavazza, L., Mauri, M.: A Formalization of UML Statecharts for Real-Time Software Modeling. The 6th Biennial World Conference On Integrated Design Process Technology (IDPT 2002), "Towards a rigorous UML" session, Pasadena (2002)

8. Eshuis, R., Wieringa, R.: Requirements-Level Semantics For UML Statecharts. Formal Methods for Open Object-Based Distributed Systems - FMOODS'2000, Stanford (2000)

9. Ghezzi, C., Mandrioli, D., Morzenti A.: TRIO, a logic language for executable specifications of real-time systems. The Journal of Systems and Software, Vol. 12, n. 2. Elsevier Science (1990)

10. Heitmeyer C.L., Jeffords R.D., Labaw B.G., Comparing different approaches for Specifying and verifying Real-Time Systems, in Proc. 10th IEEE Workshop on Real-Time Operating Systems and Software, New York (1993) 122-129

11. Kesten, Y., Pnueli, A.: Timed and Hybrid Statecharts and their Textual Representation. In Formal Techniques in Real-Time and Fault-Tolerant Systems $2^{\text {nd }}$ International Symposium (1992)

12. Lavazza, L., Quaroni, G., Venturelli, M.: Combining UML and formal notations for modelling real-time systems. In: Gruhn, V. (ed.): Proceedings of ESEC/FSE 2001, Vienna, ACM Press (2001)

13. Mauri, M.: Estensione di UML per la specifica e la verifica delle proprietà di sistemi Real Time. Politecnico di Milano, Thesis (in Italian), http://www.polimi.it (2001)

14. OMG: Unified Modeling Language Specification, Version 1.4. http://www.omg.org (2001)

15. Pnueli A., Shalev, M.: What is in a step: On the semantics of statecharts. In: Ito T., Meyer, A. R. (eds.): Intl. Conf. TACS '91: Theoretical Aspects of Computer Software. Lecture Notes in Computer Science, Vol. 526. Springer-Verlag, Berlin Heidelberg New York (1991) 244-264

16. Rational Software Corporation: Modeling Language Guide, Rational Rose ${ }^{\circledR}$ RealTime, http: / / www . rational.com (2002)

17. Reggio, G., Astesiano, E., Choppy C., Hussmann, H.: Analysing UML Active Classes and Associated State Machines-A Lightweight Formal Approach. In Maibaum, T. (ed.): Proc. FASE 2000- Fundamental Approaches to Software Engineering. Lecture Notes in Computer Science, Vol. 1783. Springer-Verlag, Berlin Heidelberg New York (2000)

18. Selic, B., Gullekson, G., Ward, P. T.: Real-Time Object-Oriented Modeling. Wiley (1994)

19. Sifakis, J.: Modeling real-time systems-challenges and work directions. EMSOFT01, Tahoe City, October 2001. Lecture Notes in Computer Science, Vol. 2211. Springer-Verlag, Berlin Heidelberg New York (2001)

20. Yovine, S.: Kronos, A Verification Tool for Real-Time Systems, Kronos User's Manual Release 2.2. Springer International Journal of Software Tools for Technology Transfer, Vol. 1 October (1997) 